\title{
Magische Alltagsvorstellungen und spätmittelalterliche Zaubereiprozesse in Tirol
}

\section{Hester Margreiter}

Kerngebiet: Mittelalterliche Geschichte

eingereicht bei: Ao. Univ.-Prof. Dr. Klaus Hubert Brandstätter

eingereicht im: SS 2014

Rubrik: SE-Arbeit

\begin{abstract}
Common Magical Concepts and Late-Medieval Sorcery Trials in Tyrol

The beliefs of the people in the late Middle Ages went beyond the canonical doctrine of the Catholic Church. Magic performances complemented the religion and its application played a central role in coping with everyday life. The aim of this paper is to discuss the historical function of magical ideas in social context, their importance for everyday life and world-view, and the criminalization, legal interpretation and prosecution of "magical crimes". The conclusion tries to offer guidelines to distinguish between socially accepted magic and criminalized sorcery on the eve of the European witch hunting era.
\end{abstract}

\section{Inhalt und Fragestellung}

Die Glaubensvorstellungen der Menschen im Spätmittelalter gingen über die kanonisierte Lehre der katholischen Kirche hinaus, magische Vorstellungen ergänzten die Religion und ihre Anwendung spielte eine zentrale Rolle in der Alltagsbewältigung.

„Unter Magie wird jedes System von Vorstellungen und Verhaltensweisen verstanden, das darauf abzielt, die sichtbare, im Alltag erlebbare Welt mit einem Raum außerhalb dieser Welt in Beziehung zu setzen. Dieses System wird von Einzelnen oder informellen Kleingruppen getragen, die jeweiligen Vorstellungen und Verhaltensweisen sind weder institutionalisiert noch unterliegen sie allgemeinen fixen Regeln oder Dogmen."

1 Johannes Dillinger, Hexen und Magie. Eine historische Einführung (Historische Einführungen 3), Frankfurt/ Main 2007, S. 13. 
Die historische Funktion dieser magischen Vorstellungen im sozialen Kontext, in ihrer Bedeutung für Altag und Weltsicht, sowie ihre Kriminalisierung und juristische Interpretation sind zentrale Untersuchungsgegenstände dieser Arbeit. Des Weiteren wird die Verfolgung von Menschen, welchen ein "crimen magiae" vorgeworfen wurde, behandelt.

Das einleitende Kapitel „Begriffe und Begrifflichkeiten“ widmet sich der Diskussion um die passende wissenschaftliche Diktion und damit auch der Frage nach einer möglichen schichtspezifischen Abgrenzung historischer magischer Vorstellungen. Weiters wird auf die wissenschaftlichen Quellen und die historische Terminologie eingegangen und eine Definition für Magie im Vergleich zu Religion angeboten. Das folgende Kapitel „Von gutem und bösem Zauber" soll einen Überblick über Wesen und Funktion der Alltagsmagie, ihre Rezeption und Bekämpfung durch religiöse Eliten und ihren Eingang in die Strafprozessordnung bieten. Dabei wird versucht die Subjektivität und versuchte Objektivierung in der Trennung zwischen allgemein gebräuchlichen Zaubermitteln und schädlicher Magie nachzuzeichnen. Dies passiert in Abgrenzung zu späteren Hexereivorstellungen, die nicht Gegenstand dieser Arbeit sind und daher nur am Rande und zur Unterscheidung behandelt werden. Interessant in diesem Zusammenhang ist, dass magische Praktiken nicht nur bekämpft, sondern auch zur Verfolgung und Abwehr von "Hexen" angewandt wurden. Die konkreten Vorstellungen und angewandten Praktiken waren von erstaunlicher Vielfalt, da Magie in jedem Lebensbereich eingesetzt werden konnte. Daher bietet dieses Kapitel nur einen Überblick über die häufigsten Formen der Alltagsmagie.

Die „Pluemen der Tugent“ stellen die Übersetzung eines älteren italienischen Textes dar, welcher durch eine Auflistung alltagsmagischer Vorstellungen des frühen 15. Jahrhunderts ergänzt wurde. In Kombination mit der wissenschaftlich aufgearbeiteten Überlieferung der Tiroler Zauberei- und Hexenprozesse bietet sich damit eine Möglichkeit, die Thematik regionalspezifisch zu untersuchen. Zusätzlich bietet die Schrift einige zeitgenössische Erklärungsvorschläge zur Herkunft magischer Vorstellungen, da es sich um eine Kritik des Magieglaubens handelt.

Das letzte Kapitel über die „Zaubereiprozesse in Tirol“ bietet einen Überblick über juristisch verhandelte Magievorstellungen vom 13. Jahrhundert bis zur Mitte des 16. Jahrhunderts. Am Ende dieser Zeitspanne findet sich ein etwa fünfzigjähriger Zeitraum, in dem neben Zauberei- auch die ersten Hexereiprozesse stattfanden. Mit Schwerpunkt auf den Innsbrucker Prozess 1485 und unter Berücksichtigung des obrigkeitlichen Verhaltens sollen vor allem hinsichtlich der Strafurteile und des Geschlechts der Beschuldigten Unterschiede zwischen Zauberei- und Hexereiprozessen herausgearbeitet werden.

Als Forschungsfrage soll behandelt werden, unter welchen Umständen Magie als verwerflich betrachtet wurde, und es soll eine Abgrenzung von kirchlich sowie obrigkeitlich tolerierten versus verfolgten magischen Vorstellungen und Handlungen versucht werden. Dabei sollen auch Unterschiede und Gemeinsamkeiten von Zaubereiund Hexereiglauben hinsichtlich der juristischen Verfolgung herausgearbeitet werden. 


\section{Begriffe und Begrifflichkeiten Magie, Aberglaube oder Volksglaube?}

Der Begriff „Aberglaube“, lat. superstitio, war ein zeitgenössischer Sammelbegriff, mit dem alle von der Amtskirche verurteilten Formen magischer Vorstellungen und Praktiken des Spätmittelalters und der Neuzeit zusammengefasst wurden. Um die negative Konnotation des Begriffs "Aberglaube“ zu vermeiden, wurde in der neueren Fachliteratur über magische Alltagsvorstellungen vielfach der Begriff "Volksglaube“ verwendet. ${ }^{2}$

Dieser Begriff ist insofern problematisch, als er impliziert, dass diese Vorstellungen ausschließlich im "gemeinen Volk” zu finden gewesen wären. Der Glaube an magische Praktiken und deren Durchführung war jedoch in allen Teilen der Bevölkerung, von Besitzlosen über die bäuerlich und handwerklich tätigen Menschen bis hin zu Adeligen und der Hofgesellschaft, verbreitet. Hinsichtlich der magischen Vorstellungen eine Grenze zwischen Volks- und Elitenglaube ziehen zu wollen, würde der gelebten Praxis widersprechen. Zeitgenössische Quellen berichten davon, dass dieselben Frömmigkeitsformen in den verschiedenen Sozial- und Bildungsschichten oftmals mit gleicher Emotionalität gelebt wurden. ${ }^{3}$

Die ideologische Konnotation des Begriffs Volksfrömmigkeit stammt aus dem Zeitalter der Aufklärung, da sich die gebildeten Schichten des 18. und 19. Jahrhunderts von den Glaubensvorstellungen der einfachen Landbevölkerung distanzieren wollten. Volksfrömmigkeit sollte als unreflektierte Massenreligiosität mit abergläubischer Durchdringung von einer Elitenfrömmigkeit, welche "rational“ durch das Bibelstudium untermauert wurde, abgegrenzt werden. Die weitere ideologische Konnotation des Begriffs Volksfrömmigkeit als Ausdruck einer unterstellten Homogenität des gesamten Volkes, unter Missachtung unterschiedlicher Lebensrealitäten aufgrund der Verteilung von Macht, Ehre und Reichtum in der ersten Hälfte des 20. Jahrhunderts, belastet somit auch den Werkzeugcharakter dieses Begriffs. ${ }^{4}$

Magische Vorstellungen waren vielfach mit kirchlichen Symbolen, Riten und Vorstellungen verknüpft. Dies betrifft beispielsweise Wettersegen, Reliquienverehrung oder Kräuterweihe. Vorstellungen wundertätiger Bilder, blutender Hostien oder religiöser Visionen wurden von der Amtskirche in manchen Fällen unterstützt, in anderen nach Möglichkeit unterdrückt. Wallfahrten zu wundertätigen Orten waren oftmals Massenbewegungen spätmittelalterlicher Laien. Die synkretistische Qualität des spätmittelalterlichen Alltags, d.h. die Symbiose zwischen magischen und kirchlichen Glaubensvorstellungen bezieht sich neben den bäuerlichen Gemeinschaften ebenso auf die bürgerlichen, klerikalen und adeligen Schichten, welche diesen Glaubensvorstellungen in unterschiedlicher, aber ähnlicher Form nachhingen. Neben der Qualität religiöser Alltagsvorstellungen sollte aber auch ihre Funktion untersucht werden und daher an dieser Stelle Max Webers Analyse nicht unerwähnt bleiben: Sowohl magischer als auch kirchlicher Glaube zielten

2 Dillinger, Hexen und Magie, S. 18.

3 Klaus Schreiner, Laienfrömmigkeit - Frömmigkeit von Eliten oder Frömmigkeit des Volkes? Zur sozialen Verfaßtheit laikaler Frömmigkeitspraxis im späten Mittelalter, in: Laienfrömmigkeit im späten Mittelalter. Formen, Funktionen, politisch-soziale Zusammenhänge (Schriften des Historischen Kollegs, Kolloquien 20), hrsg. von Klaus Schreiner, München-Oldenbourg 1992, S. 1-78, hier S. 6.

4 Schreiner, Laienfrömmigkeit, S. 7. 
schichtenübergreifend auf eine Erlösungsrealität. Sie zielten in den unterprivilegierten Schichten überwiegend auf die Grundlagen der menschlichen Existenz wie Gesundheit, Nahrungsversorgung, Fortpflanzung und Sicherheit, hatten in den ökonomisch privilegierten Schichten aber auch die Aufgabe, sinnstiftend und legitimierend zu wirken. ${ }^{5}$ Klaus Schreiner fasst wie folgt zusammen:

„Dem Begriff Volksfrömmigkeit liegt die Annahme zugrunde, volkstümliche Religiösität sei als Korrelat schichtgebundener Erwerbs- und Soziallagen zu begreifen. Dem widerspricht der Befund der Quellen. Volksfrömmigkeit ist nicht Frömmigkeit einer sozial homogenen Trägergruppe, sondern Medium und Ausdrucksform ständeübergreifender religiöser Homogenität. “6

Auch eine zeitgenössische Quelle widerlegt die These von magischem Glauben und Praktiken als Schichtenspezifikum. So schrieb Paracelsus (Theophrastus Bombastus von Hohenheim) im Vorwort zu seinem 1530 erschienenen Werk "Die große Wunderartzney“, bei welchen Leuten er Erkundigungen über (magische und nichtmagische) Heilpraktiken eingeholt habe:

"[Er habe] in allen den enden un orten fleissig und embsig nachgefragt / Erforschung gehabt gewisser un erfarner wahrhafften Künsten der Artzney / nit allein bey Doctorn / sondern auch bey den Scherern / Badern / gelehrten Artzten / Weibern / Schwartzkünstlern / so sich deß pflegen / bey den Alchimisten / in Klöstern / bey den Edlen und Unedlen / bei den gescheuden und eynfeltigen [...]."

Trotz der Verflechtungen zwischen Magie und Religion kann eine gewisse Abgrenzung getroffen werden. Im Folgenden sollen die wichtigsten von Johannes Dillinger zusammengefassten Merkmale genannt werden: So geht Magie von Gesetzmäßigkeiten aus und versucht mittels Ähnlichkeit bzw. "Sympathie“ und Kontakt zu Dingen und Lebewesen eine Wirkung zu erzielen. Diese "Sympathiezusammenhänge” konnten Mineralien, Tiere, Pflanzen, Körperteile, Gegenstände und Planeten umfassen und durchaus komplexen Charakter annehmen. Laut James George Frazer, einem der Väter der Kulturanthropologie, ist der große Unterschied in der jeweiligen Erwartungshaltung zu sehen: Während es bei der Ausübung von Religion darum gehe, dass höhere Mächte an sie herangetragene Wünsche freiwillig erfüllen, würden magische Handlungen Manipulationen mit dem Anspruch auf (quasi natur-)gesetzliche Wirksamkeit darstellen. ${ }^{8}$ Nach Émile Durkheim und Marcel Mauss können in der Soziologie die beiden Systeme insofern unterschieden werden, als Religion eine institutionalisierte Glaubensgemeinschaft mit fixierten abstrakten Aussagen unter staatlicher Förderung darstellt, während Magie informell von Einzelpersonen oder in kleinen Gruppen praktiziert und von staatlicher Seite

5 Schreiner, Laienfrömmigkeit, S. 2-5.

6 Ebd., S. 10.

7 Theophrastus von Hohenheim, Die große Wundartzney, Frankfurt 1530, Vorwort, zit. nach Helmut Nemec, Zauberzeichen. Magie im volkstümlichen Bereich, Wien 1976, S. 36.

8 Dillinger, Hexen und Magie, S. 14. 
bestenfalls geduldet wird. ${ }^{9}$ Mauss lehnt allerdings eine ganz strikte Trennung zwischen Religion und Magie entschieden ab.

\section{Quellen und Terminologie}

Als Quellen für magische Altagsvorstellungen existieren neben Gerichtsakten, diesbezüglichen Korrespondenzen und Flugblättern auch Traktate und Bußbücher. Diese Quellen lassen sich für sich genommen jedoch kaum als Tatsachenberichte lesen, sondern müssen mit anderen Quellen verglichen werden. Gerade Bußbücher bieten zwar regelrecht enzyklopädische Listen über magische Vorstellungen, sind aber innertheologischen Argumentationstraditionen verhaftet und können insofern einen Schwerpunkt auf von Seiten der Amtskirche verurteilte Praktiken beinhalten. ${ }^{10}$ Christliche Bußbücher des 5. bis 9. Jahrhunderts sind aber jedenfalls Belege für die Vermischung von magischen und christlichen Vorstellungen. Neben Zauberei wurden auch Wahrsagerei, Traumdeutung und Geister genannt: Die Zauberer wurden dabei in drei Gruppen eingeteilt: "malefic" (Schädiger), "tempestarii" (Wettermacher) und "venifici" (Giftmischer). "

Probleme bei der Klassifizierung von Zauberei- und Hexereiprozessen ergeben sich durch eine nicht mit unserem heutigen Sprachgebrauch übereinstimmende Terminologie. In den „österreichischen“ Ländern war der Terminus Hexe nicht gebräuchlich. Stattdessen wurden in deutschsprachigen juristischen und weltlichen Quellen die Begriffe "Unholdin" bzw. "Zauberer" und "Zauberin“ verwendet. Die häufigsten lateinischen Begriffe waren zunächst "striga” und später (und häufiger) "malefica“. So könnten die beiden "strigae“, welche im Jahr $1296^{12}$ in Tirol verbrannt wurden, sowohl "Schadenszauberinnen" als auch Giftmörderinnen gewesen sein. Jedenfalls kann nicht von einer einheitlichen Terminologie ausgegangen werden, vielmehr war eine Vielzahl an Bezeichnungen in Verwendung. Die Vorstellungen und Phantasien der theologischen Dämonologie sind besser überliefert, doch bestand auch eine volkstümliche Dämonologie, deren Inhalte allerdings schwerer und nur unvollständig zu bestimmen sind. ${ }^{13}$

Magie und Zauberei galten in der Alltagskultur des Mittelalters nicht als Sünde oder schweres Vergehen, sondern waren Alltäglichkeit und legitimes Mittel um sich zu schützen, verletzte Ordnungen wiederherzustellen und seine Rechte zu behaupten. Ziel oder Zweck der Zauberei und magischen Handlungen waren nicht nur die Abwehr des Bösen (Truten, Unholde etc.), Segnungen und Beschwörungen, sondern auch das Lösen alltäglicher

9 Dillinger, Hexen und Magie, S. 16-17.

10 Ebd., S. 10-11 u. 29.

11 Hansjörg Rabanser, Hexenwahn. Schicksale und Hintergründe. Die Tiroler Hexenprozesse, InnsbruckWien 2006, S. 13 und Hansjörg Rabanser, Die Hexenverfolgungen in Tirol. Verlauf, Prozessbiographien, Interpretation, Diss. Innsbruck 2005, S. 27, vgl. auch Wolfgang Behringer, Hexenverfolgung in Bayern. Volksmagie, Glaubenseifer und Staatsräson in der Frühen Neuzeit, München 1987, S. 71.

12 Anm. der Verfasserin: Im Fließtext bei Heide Dienst (siehe folgende Fußnote) steht die Jahreszahl 1292, bei Vergleich mit der dazugehörigen Fußnote und anderen Autoren (z.B. Rabanser) ergibt sich die Schlussfolgerung, dass es sich dabei um einen Tippfehler handelt.

13 Heide Dienst, Magische Vorstellungen und Hexenverfolgungen in den österreichischen Ländern (15. und 18. Jahrhundert), in: Wellen der Verfolgung in der österreichischen Geschichte, hrsg. von Erich Zöllner (Schriften des Institutes für Österreichkunde 48), Wien 1986, S. 70-96, hier S.76-77. 
Probleme: So sollte ein Stück vom Holz eines Galgens im Bett Wanzen vertreiben oder bei richtiger Anwendung ein einschlägiger Spruch zukünftige Spielschulden vermeiden. ${ }^{14}$

Die Delikte des Teufelspakts, der Teufelsbuhlschaft, des Hexenfluges und-sabbats sind keine Kennzeichen von Zauberei, sondern gehören in Verbindung mit dem Schadenszauber, welcher für sich genommen dem klassischen magischen Vebrechen entspricht, zum elaborierten Hexenbegriff ${ }^{15}$. Nach Hansjörg Rabanser setzt Zauberei, nicht immer, aber meist, die Absicht eines Schadens voraus, er definiert wie folgt:

"Zauberei (crimen magiae) ist die bewusste Anwendung geheimer und abergläubischer Mittel, Praktiken, Sprüche und sonstiger Künste, die man selbst erlernt oder durch die Hilfe einer anderen Person oder eines Dämons erhalten hat. Die Zauberei bringt Wirkungen hervor, welche die gewöhnlichen Kräfte eines Menschen übersteigen. [...] Einzig und allein das Delikt des Schadenszaubers ist von Bedeutung. Eine Zauberin oder ein Zauberer bedienen sich magischer Praktiken, Segnungen und Sprüche, um eine für den Menschen unmögliche Tat zu vollführen, welche entweder positive oder negative Folgen hat." ${ }^{16}$

\section{Von gutem und bösem Zauber}

\section{Magische Alltagspraktiken}

Im Hochmittelalter waren neben kirchlichen Ritualen auch "magische“ Praktiken fest in der Gesellschaft verankert. Die Menschen glaubten an günstige Magie wie Heil- und Segenszauber und fürchteten sich vor schädlicher Magie, vor Schadenszauber. Wem die Macht zu zaubern zugeschrieben wurde, der konnte in der landläufigen Meinung sowohl heilen als auch schaden. Zauber und Gegenzauber, Heil- und Schadenszauber wurden als Teil der Lebensrealität betrachtet. ${ }^{17}$ Personen, die im Sinne des klassischen, elaborierten Hexenbegriffs tatsächlich tätig wurden, gab es sicherlich nicht, jedoch zahlreiche Personen, die an eigene und fremde zauberische Fähigkeiten und Wirkungen glaubten. Diese magische Kultur wurde von Menschen aller Stände selbst angewandt und sie griffen dabei auch auf "kundige“ Personen zurück. ${ }^{18}$ Magie und Zauberei waren zunächst kein Vergehen, sondern legitimes Mittel, um sich zu schützen und verletzte Ordnung wiederherzustellen. Auch der Schutz vor Zauberei beinhaltete zauberische Sprüche und Praktiken, was allgemein akzeptierte Normalität darstellte. ${ }^{19}$

14 Rabanser, Hexenwahn, S. 15.

15 Bis zur ersten Hälfte des 15. Jahrhunderts wird der charakteristische Hexenbegriff argumentativ in zahlreichen Niederschriften vorbereitet und bis ins 16. Jahrhundert hinein weiterentwickelt und verankert. Die einflussreichsten Schriften waren der „Formicarius" (ca. 1437) des Johannes Nider, ein Überblick über die vorangegangenen Schriften zum Thema der Hexerei, und der "Malleus Maleficarum" (Erstdruck 1517) des Heinrich Institoris. Vgl. Rabanser, Die Hexenverfolgungen in Tirol, S. 29.

16 Rabanser, Die Hexenverfolgungen in Tirol, S. 22-23.

17 Dienst, Magische Vorstellungen, S.70-71.

18 Rabanser, Die Hexenverfolgungen in Tirol, S. 376

19 Rabanser, Hexenwahn, S. 15. 
Bei Verdacht auf einen Schadenszauber, aufgrund unerklärlicher Schäden oder besonderer Vorzeichen und Begleiterscheinungen, wurde zunächst ein potentiell Schuldiger ausgemacht und versucht, diesen zur Rücknahme oder Wiedergutmachung des Schadens zu bewegen. Wenn dies nicht erfolgreich war, war der nächste Schritt die Anwendung eines Gegenzaubers, auch mit Hilfe von „kundigen“ Personen, wie Heilern, Zauberbannern, Wahrsagern, Hebammen oder Scharfrichtern. Hier galt der Leitsatz "similia similibus curantur", also Gleiches mit Gleichem zu heilen bzw. zu bekämpfen. Neben der Bekämpfung des Schadens hatte der Gegenzauber auch die Funktion eines Gottesurteils, der Täter sollte bloßgestellt werden, indem er körperlich oder geistig geschädigt wurde. Beispielsweise sollte das Umhertragen von Diebesgut zu Schmerzen und Bewegungsproblemen führen. Ein Gegenzauber war ein legitimes Mittel in der magischen Alltagskultur, die (geistliche) Obrigkeit sah jedoch darin ein Verbrechen. Heinrich Institoris ${ }^{20}$ schrieb 1486 im Hexenhammer:

„Es zeigt sich auch, dass [Behexte] sehr selten befreit werden, sofern sie göttliche Hilfe und den Beistand der Heiligen anflehen: Folglich können sie nur durch die Hilfe von Dämonen befreit werden. Diese jedoch zu suchen, ist nicht erlaubt."21

Vergrabene oder versteckte Zauberutensilien, ebenso wie verschüttete Flüssigkeiten, wurden als Schadenszauber an Orten versteckt, die mit der zu schädigenden Person in enger Verbindung standen. Besonders beliebt in diesem Zusammenhang war der Schwellenzauber, bei dem an der Haustür, unter der Schwelle oder beim Stalltor ein Gegenstand deponiert wurde. So berichtete im Innsbrucker Hexenprozess eine Zeugin, die sich als Opfer eines angehexten Leidens betrachtete, vom Auffinden einer mit Nadeln durchstochenen Wachspuppe unter der Schwelle ihrer Haustür. ${ }^{22}$

Die Anwendung von Heilmitteln erfolgte häufig in Verbindung mit Magie, aber oft wurde auch lediglich auf die Wirksamkeit eines Heilzaubers vertraut. Heilungsmagie wurde gegen körperliche Beschwerden, Krankheiten und Verletzungen, aber auch für die Gesundheit des Viehs oder ökonomischen Erfolg im Allgemeinen angewandt. Heilungsmagie wurde häufig in Form eines Transferzaubers ausgeübt, dafür wurde beispielsweise ein magischer Gegenstand am erkrankten Körperteil gerieben, wodurch die Krankheit darauf übergehen und mit der Zerstörung des Gegenstandes dieser auch als angenommener Krankheitsträger vernichtet werden sollte. Häufig wurde Heilungszauber aber auch in Verbindung mit medizinisch durchaus wirksamen Pflanzen angewandt, beispielsweise durch einen magischen Spruch oder ein Ritual während der Herstellung einer Arznei. ${ }^{23}$

Ein weiteres beliebtes Anwendungsgebiet der Altagsmagie war die Herstellung und Sicherung von Liebesbeziehungen. Eine lateinische Beschwörungsformel für Liebeszauber, enthalten im Codex 960 der Universitätsbibliothek Innsbruck, datiert Ende des 14. Jahrhunderts, verbindet alltagsmagische Vorstellungen mit christlichen Formeln. Mithilfe von Eisenkraut (verbena) soll ein Liebeszauber ermöglicht werden. Die Pflanze musste

20 Es sei angemerkt, dass Insistoris selbst für seine Zeit eine extreme Haltung an den Tag legte.

21 Zit. n. Rabanser, Hexenwahn, S. 18-19.

22 Rabanser, Die Hexenverfolgungen in Tirol, S. 476-477.

23 Dillinger, Hexen und Magie, S. 32-33. 
dafür vor Sonnenaufgang unter litaneiartiger Anrufung beschafft werden. Einleitend sollten folgende Worte gesprochen werden:

„Im Namen des Vaters und des Sohnes und des heiligen Geistes Amen. Verbena, voll der Gnade Gottes [...] ich bitte dich darum, dass du mich erhörst [...]"

Die anschließende eigentliche Beschwörung lautet:

„Ich beschwöre dich [...], dass du solche Macht habest, dass du jedem Menschen, den du berührt hast, sei es Mann oder Frau, durch alle Kräfte und durch alle vorgesagten derartigen Beschwörungen so machst, dass, wer immer es sein mag, er mich lieben und mich wie Gott nicht wechseln könne und dass du dieses selbe bewirkst, wem du das in Speise und Trank gibst."

Es folgt eine Anweisung:

„Inzwischen nimm sie (die Pflanze) und sprich: Vater (unser) und Ave Maria und gib alle in Speise und Trank oder berühre den nackten Körper und er (sie) wird dich sicher lieben." 24

Beliebte Vorstellungen waren auch der magische Diebstahl von Milch oder Wein. Bei Milch wurde zumeist ein Baum oder Stück Holz angebohrt und daraus die Milch entnommen, welche dann einem klar benennbaren Hof gefehlt habe. Wein wurde zumeist durch den magischen Einbruch in Keller entwendet. Dabei wären Fässer ausgetrunken und anschließend auf zaubrische Art und Weise mit minderwertigem Wein oder Urin und Fäkalien wiederaufgefüllt worden. Eintritt in einen Stall bzw. die Möglichkeit zu einer „Kellerfahrt" ermöglichte der Teufel. Daher ist diese Vorstellung des Schadenszaubers schon in den Bereich der Hexerei zu rechnen. ${ }^{25}$

In der Alltagsmagie wurden Dämonen nur selten angerufen. Häufiger wurde Hilfe von Gott, Engeln oder Heiligen erbeten. Daneben spielten Geister eine wichtige Rolle. Naturund Hausgeister standen in direkter, meist positiver Beziehung zum Alltagsgeschehen. Ihnen wurde die Beeinflussung des Wetters oder der Gesundheit des Viehs zugeschrieben. Unsichtbare helfende Geister waren direkt aktiv, als Dank wurden rituell Speisen dargeboten. Diese Geister hatten auch symbolischen Wert bzw. disziplinierenden Charakter, indem das Nicht-Einhalten des Verhaltenskodex mit Bestrafung durch Hausgeister bedroht wurde. Totengeister galten als spukende Verstorbene, die keine Ruhe finden konnten, was als schweres Unglück galt. Geschichten über Verstorbene, die aufgrund unrechtmäßiger Taten als Totengeister wiederkehrten, hatten ebenfalls disziplinierenden Charakter. In der katholischen Kirchenlehre war die Wiederkehr von Seelen aus dem Fegefeuer, welche die Lebenden mahnen sollten, nicht zu sündigen, nicht endgültig ausgeschlossen. Daher wurde dieser Glaube von den Geistlichen auf lokaler Ebene nie kategorisch abgelehnt. Auch wenn im reformatorischen Glaubenskodex die Totengeister eine Unmöglichkeit

24 Codex 960, ULB Innsbruck, zit. nach Rabanser, Hexenwahn, S. 36.

25 Ebd., S. 177. 
darstellten, wurden sie dennoch auch von protestantischen Obrigkeiten akzeptiert. Daraus lässt sich die tiefe Verankerung des Totengeisterglaubens ermessen. ${ }^{26}$

Eine breite Anwendung fanden magische Zeichen an Gebäuden, Werkzeugen oder Möbeln. Diese sollten genauso wie Amulette einen passiven Schutz verleihen. Amulette als unheilabwendende Kraftgegenstände konnten Edelsteine, pflanzliche oder tierische Überreste, rote Fäden oder auch Bruchstücke von Reliquien beinhalten. Eine der einfachsten Formen war das Zettelamulett, bei welchem ein auf einem Stück Papier notierter Segensspruch am Körper getragen wurde. Segenssprüche sind ein Beispiel für das Nebeneinander und die praktische Vermengung von kirchlichen Riten und magischen Alltagspraktiken. Ein Kreuzzeichen oder die Anrufung der Dreifaltigkeit z.B. waren ganz klar ein Teil der katholischen Frömmigkeit und wurden zusammen mit Schutz-, Bannund Heilungssprüchen magischer Natur angewandt. Magische Sprüche nahmen oft auf Geschichten Bezug, in denen Heilige oder sogar Jesus selbst mit einem ähnlichen wie dem zu lösenden Problem konfrontiert waren. ${ }^{27}$

Die Alltagsmagie stellte also eine Ergänzung katholischer Frömmigkeit dar und war nicht nur von magischen und okkulten Elementen geprägt, sondern vermischte sich auch mit liturgischen Praktiken und Sprüchen. So wurden sakrale Gegenstände oftmals mit magischen Praktiken gekoppelt, da man an eine Wirkungsübertragung glaubte. Beispielsweise wurde Weihwasser auch magisch verwendet oder Gegenstände unter das Altartuch gelegt. Die Vermischung von Alltagsmagie und kirchlicher Praxis wurde von der Amtskirche geduldet und teilweise auch unterstützt. ${ }^{28}$ Heiligenverehrung wurde in diesem Zusammenhang als „do-ut-des” betrieben, also mit der Vorstellung, dass der Heilige bei korrekter Anrufung die gewünschte Wirkung zu leisten hatte. Eine derartige Wirkungslogik war in der offiziellen Lehre der Kirche nicht vorgesehen. Ein Ausbleiben dieser Wirkung wurde aber bisweilen auch mit der "Bestrafung" durch Beschimpfung oder "Misshandlung" einer Heiligenstatue geahndet. ${ }^{29}$ In manchen Bereichen wurde die Vermengung von magischen Vorstellungen und kirchlichen Traditionen jedoch in die kanonisierte Lehre der katholischen Kirche aufgenommen, hierbei sei vor allem auf die Reliquienverehrung hingewiesen. Der Glaube an die Wirksamkeit von Reliquien konnte bisweilen große Menschenmengen mobilisieren, so wurde die Reliquiensammlung des Ritters Florian Waldauf, welche 1501 feierlich in die Haller Pfarrkirche überstellt wurde, jährlich im Rahmen einer Heiltumschau von großen Menschenmengen besucht. Laut einer Urkunde Julius II. von 1509 nahmen an einer Heiltumschau rund 10.000 Menschen teil. ${ }^{30}$

Ab dem 16. Jahrhundert wurde im Kampf gegen die reformatorischen Bewegungen ein Wandel eingeleitet und ein Teil jener Praktiken und Rituale, die von der protestantischen Kritik bevorzugt angegriffen wurden, verdrängt, als sündhaft umgedeutet und sogar

26 Dillinger, Hexen und Magie, S. 38-40.

27 Ebd., S. 33-34.

28 Rabanser, Hexenwahn, S. 16.

29 Dillinger, Hexen und Magie, S. 35.

30 Stefan Handle, Religiöser und gesellschaftlicher Wandel in Imst in der frühen Neuzeit (Schlernschriften 357), Innsbruck 2013. 
dämonisiert. Eine Zäsur stellte hier das Konzil von Trient 1545 bis 1563 dar, welches auch tradierte und häufig ausgeübte Altagspraktiken als "gotteslästerliche Zauberei" brandmarkte. Dies war ein disziplinierendes und kriminalisierendes Moment. ${ }^{31}$ Die diesbezüglichen Ansichten übertrugen sich auch auf die Bevölkerung und hatten eine breite Sensibilisierung und Wahrnehmungsverzerrung zur Folge, welche Mutmaßungen und Denunziationen verstärkte und eine praktische Möglichkeit bot, störende Menschen aus der Gesellschaft zu eliminieren. ${ }^{32}$

\section{Zauberei aus theologischer Sicht}

Der "Formicarius" des Johannes Nider ist eine in der ersten Hälfte des 15. Jahrhunderts entstandene theologische Abhandlung, welche auch Magie und Zauberei behandelt. Das fünfte Buch des „Formicarius", welches den Titel "Von den Zauberern und ihren Täuschungen" trägt ${ }^{33}$, definiert wie folgt:

„Als ein Zauberer (maleficus) wird nämlich jemand bezeichnet, der Schlechtes tut (malefaciens) oder schlecht dem Glauben dient (male fidem servans). Beides findet man häufig bei den Zauberern, die durch abergläubische Handlungen (maleficorum supersticiones) ihre Mitmenschen verletzen. “34

Weiters wird ausgeführt, dass Zauberer Menschen nur unter Zulassung Gottes Inunquam nisi deo permittente) schädigen würden. Das unmittelbare Zufügen der Schäden würde dann von den Dämonen bewerkstelligt werden. Die Zauberer würden mittels Worten, Riten und Handlungen diesbezügliche „Quasi-Pakte“ mit Dämonen eingehen. Es werden sieben Arten von Zauberei aufgezählt:

"1.) das Hineintragen der "schlechten Liebe" (amorem malum) bei einem Mannes [sic!] oder bei einer Frau, 2.) das Erzeugen von Haß oder Neid in einem Menschen, 3.) die Erzeugung von Impotenz beim Mann oder Unfruchtbarkeit bei der Frau, d. h. bei denen, die "Verhexte" (malificati) genannt werden, 4.) das Krankmachen eines Menschen an einem Körperteil, 5.) die Vernichtung von Leben, 6.) der Raub des Verstandes, 7.) die wirksame Schädigung eines Menschen in seinen Angelegenheiten oder seinem Denken mit den genannten Mitteln. ${ }^{\text {35 }}$

Der 1486 verfasste "Malleus maleficarum“ oder Hexenhammer des Inquisitors Heinrich Institoris war insofern prägend, als die Schrift eine der ersten Dämonologien war, welche mithilfe des Buchdrucks rasch verbreitet wurde. Das Verwenden der lateinischen Sprache machte das Buch für Juristen und Theologen gut benutzbar und war vor allem in den deutschen Ländern von unmittelbarer Bedeutung. Wolfgang Behringer schätzt, dass allein bis 1523 rund 10.000 Exemplare gedruckt wurden. ${ }^{36}$ Die Kernaussage, dass Hexen

31 Rabanser, Hexenwahn, S. 17.

32 Ebd., S. 18.

33 Werner Tschacher, Der Formicarius des Johannes Nider von 1437/38. Studien zu den Anfängen der europäischen Hexenverfolgungen im Spätmittelalter, Aachen 2000, S. 389.

34 Zit. n. Ebd., S. 389.

35 Zit. n. Ebd.

36 Wolfgang Behringer, Heinrich Kramers "Hexenhammer": Text und Kontext, in: Frühe Hexenverfolgung in 
ihre Schäden tatsächlich verüben würden, stellte einen Bruch zur theologischen Tradition dar, entsprach aber einer durchaus verbreiteten Ansicht. Ein weiterer bedeutsamer Aspekt der Schrift ist die Behauptung, dass Frauen von Natur aus schlecht wären, da sie leichter dem Glauben abfallen und somit den überwiegenden Teil der Hexerei und Zauberei ausübenden Personen ausmachen würden. ${ }^{37}$ Frauen wurde auch in früheren theologischen Schriften eine größere Sündenanfälligkeit und damit ein quasi angeborener, die Gesellschaft bedrohender Defekt unterstellt. Jedoch spitzt Institoris diese misogynen Behauptungen weiter zu, denn er bezieht seine Ausführungen zu Schadenszaubern überwiegend auf das weibliche Geschlecht und leitet daraus eine besondere Anfälligkeit für die Anfechtungen des Teufels ab. In seinen Forderungen geht er noch einen Schritt weiter und vollzieht den Schritt von einem Disziplinierungs- hin zu einem Tötungsprogramm, der in den von inm verarbeiteten theologischen Vorläufern nicht enthalten ist. ${ }^{38}$ Da die Verfolgung von "Hexen“ ein großes persönliches Anliegen ${ }^{39}$ von Institoris war, dürfte das gescheiterte Prozessverfahren 1485 in Innsbruck den endgültigen Anstoß für das Verfassen des Buches gegeben haben. Er wollte der weltlichen und geistlichen Obrigkeit sowie der Bevölkerung Hexerei als „Tatbestand“ und Gefahr möglichst überzeugend nahebringen und Verfolgungen anstoßen. ${ }^{40}$

\section{Juristische Handhabung der Zauberei}

Zwischen Zauberei und Hexerei wurde insofern unterschieden, als bei Zauberei, dem "crimen magiae", abergläubische Mittel, Praktiken, Sprüche oder sonstige Künste angewandt wurden. Einzig mögliches Delikt war hier der Schadenszauber. Demgegenüber lauteten die fünf klassischen Delikte, die ab dem 15. Jahrhundert Hexerei charakterisierten, Teufelspakt, Teufelsbuhlschaft, Hexenflug, Hexensabbat und Schadenszauber. ${ }^{41}$ Nach der Untersuchung von Hansjörg Rabanser zu 241 Hexerei- und Zaubereiprozessen im Raum des heutigen Nord-, Ost- und Südtirol vom 13. bis 18. Jahrhundert, wobei sich ein Großteil der Quellen auf die Zeit zwischen 1485 und 1760 bezieht, können 70\% als Zauberei- und nur 30\% als Hexereiprozesse mit zumindest einem weiteren Hexereidelikt klassifiziert werden. Rabanser sieht den Beginn der Hexen- und Zaubererverfolgung in den letzten zwei Jahrzehnten des 15. Jahrhunderts. Nach dem Innsbrucker Hexenprozess von 1485 fanden außerhalb der drei großen Prozesswellen 1501 bis 1510, 1580 bis 1645 und 1675 bis 1685 auch einzelne diesbezügliche Prozesse statt. ${ }^{42}$

Seit 1240 ging die Inquisition gegen Zauberei vor, die weltlichen Gerichte verfolgten Anschuldigungen dazu ab 1304. Die ersten Verfolgungen erfolgten noch vereinzelt und zunächst fast ausschließlich im südfranzösischen Raum. Die Inquisitionsverfahren

Ravensburg und am Bodensee (Historische Stadt Ravensburg 2), hrsg. von Andreas Schmauder, Konstanz 2001, S. 83-124, hier S. 83-84.

37 Rabanser, Hexenwahn, S. 44.

38 Behringer, Heinrich Kramers "Hexenhammer", S. 88-89.

39 Es ist nicht verwunderlich, dass bereits einige zeitgenössische Gegner Institoris als einen psychisch abnormen Menschen bezeichneten. Vgl. Ebd., S. 89.

40 Ebd., S. 45.

41 Ebd., S. 20.

42 Rabanser, Die Hexenverfolgungen in Tirol, S. 527-528. 
unterschieden sich von den weltlichen vor allem dadurch, dass hier schon deutliche Züge des späteren Hexenbildes beobachtet werden können, während weltliche Gerichte einzelne Motive der Zauberei verhandelten. In Tirol dürfte die Verfolgung überwiegend erst nach dem Verfassen der einschlägigen Schriften eingesetzt haben, da bspw. in Hans Vintlers "Pluemen der Tugent“ von 1411 lediglich literarische Vorlagen behandelt werden, der Autor, welcher selbst als Richter tätig war, sich aber nicht auf eigene Erfahrungen aus richterlicher Praxis bezieht. ${ }^{43}$

Eine reichsgesetzliche Grundlage für die Handhabung von Zaubereiprozessen erfolgte erst 1532 mit der Peinlichen Halsgerichtsordnung Karls V., der "Constitutio criminalis Carolina”. Hier wurde u.a. angedrohter und „eingetretener" Schadenszauber, das Angebot Zauberei zu lehren, die Gemeinschaft mit Zauberern und Zauberinnen bzw. ein diesbezüglicher Leumund und der Umgang mit zauberischen Worten, Dingen, Gebärden oder Weisen als Verdachtsmoment genannt. Zur „Wahrheitsfindung“ war bei fehlendem Geständnis die Folter vorgesehen, genauso wie sie bei Raub, Mord, Landesverrat u.a.m. zur Anwendung kam. ${ }^{44}$

Die landesfürstlichen Verordnungen in Tirol waren hier wesentlich zurückhaltender. In der 1499 erlassenen Halsgerichtsordnung Maximilians I. für Tirol wurde Zauberei nicht erwähnt ${ }^{45}$, genauso wenig in den Landesordnungen Ferdinands I. 1526 und 1532. In den Polizeiordnungen von 1544 und 1552 wurde Zauberei als „Fürgeben“, "Betrug” und "Aberglauben", der bestraft werden soll, bezeichnet, eine Todesstrafe wurde nicht erwähnt. Auch Maximilian II. ließ ab 1568 Zauberer und Wahrsager nicht töten, sondern öffentlich verspotten und bei Wiederholungsfällen des Landes verweisen, Ferdinand II. verhängte dafür ab 1573 lediglich Geldbußen. Erst die Neue peinliche Halsgerichtsordnung Ferdinands III. für Österreich unter der Enns von 1656 gleicht in Anweisung und Argumentation dem Hexenhammer und sah als Strafe den Tod durch Verbrennen vor, eventuell gemildert durch vorherige Enthauptung. ${ }^{46}$

\section{Die „Pluemen der Tugent“ als zeitgenössische Kritik}

\section{Hans Vintlers Leben und Werk}

Hans Vintler stand ab 1407 im Dienst Herzog Friedrichs IV. als Pfleger des Gerichts Stein am Ritten, ab 1416 als Amtmann an der Etsch und ab 1419 als Gesandter in Venedig. Er starb 1419. ${ }^{47}$ Der auf Schloss Runkelstein bei Bozen lebende Dichter übersetzte 1411 das um 1320 entstandene Werk „Fiori di virtú“, welches dem Benediktiner Tommaso

43 Franz-Josef Schweitzer, Hans Vintlers "Aberglaubensliste“ und der Hexenbegriff, in: Literatur und Sprache in Tirol. Von den Anfängen bis zum 16. Jahrhundert. Akten des 3. Symposiums der Sterzinger Osterspiele (10.12. April 1995), hrsg. von Michael Gebhardt/Max Siller (Schlernschriften 301), Innsbruck 1996, S. 281-292, hier S. 283-284.

44 Dienst, Magische Vorstellungen, S.72.

45 In der 1514 von Maximilian I. erlassenen Ordnung für die Landgerichte unter der Enns wird Zauberei allerdings verboten. Vgl. Ebd., S. 73.

46 Ebd., S.73.

47 Rabanser, Hexenwahn, S. 36. 
Gozzadini zugeschrieben wird. ${ }^{48}$ Gozzadini präsentierte darin 35 Tugenden und Laster, die einander gegenübergestellt und mit Gleichnissen und exemplarischen Geschichten ergänzt wurden. ${ }^{49}$ Vintler ergänzte die Vorlage um weitere Erzählungen, Sprichwörter, Gebete, Zitate, eine Aberglaubensliste und vieles mehr. ${ }^{50}$

In den von inm hinzugefügten Erzählungen über den alpenländischen Volksglauben beklagte Vintler die Beliebtheit von Zauberei als einem schändlichen Laster, welches er auch dem damaligen Klerus anlastete. Vintler listete eine große Menge an populären Vorstellungen auf, u.a. Teufelsbannerei (zur Hellseherei und zur Schatzsuche), das Gießen von Wachsbildern, Vogelschrei- und Traumdeutung, Liebeszaubereien, das Herstellen und Anwenden von Giften, magische Mittel zur Heilung von Krankheiten, Geistergestalten (Truten, Unholde, Orken, Elben, Schrattel), Pferde- und Schwertsegen, Zaubereien mit Körperteilen von Gerichteten, Beschwörungen und vieles mehr. ${ }^{51}$

In der 323 Verse $^{52}$ umfassenden Liste Vintlers findet meist keine Differenzierung der Phänomene nach Geschlechtern statt. Meist wird von "etleich" bzw. "etleich leut" gesprochen, welche zauberische Praktiken anwenden oder an diese glauben würden. Genausowenig versucht er eine Einteilung der unterschiedlichen Praktiken bspw. in Heilund Schadenszauber. Magische Praktiken, der Glaube an Geistergestalten oder spätere Hexenmotive wie die Verwandlung von Personen in Katzen werden undifferenziert aneinandergelistet. ${ }^{53}$

Vintler versucht eine Aufzählung der Irrtümer von Zauberei und „Aberglaube“ gemäß der Ethik und Erkenntnistheorie Thomas von Aquins. Explizite Häresien, die auf Katharer, Waldenser oder Begarden bezogen werden könnten, finden sich genauso wenig wie ein festes Bild einer "Zauberin“ oder "Hexe“, stattdessen wird auf den einen christlichen Gott verwiesen. Wolfgang Ziegeler ${ }^{54}$ vermutet ein bewusstes Ignorieren dieser Themen durch Vintler, um kein Interesse der Inquisition an seinem Amtssprengel zu wecken. ${ }^{55}$

\section{Die „Aberglaubensliste”}

Über Zauberei, magische Praktiken und Geisterglaube der Bevölkerung schreibt Hans Vintler in den „Pluemen der Tugent" wie folgt:

„[...] und das man allein got sol eren, / wann er ist chünig, chaiser ob allen herren. / aber das velscht man ietz gar ser, / wann des unglauben ist mehr, / wann sein iemant chan gesagen. / ich wais ihr vil, die da haben / ganzen

48 De Felip-Jaud, Die Sprichwörter in Hans Vintlers, Pluemen der tugent', S. 269.

49 Rabanser, Hexenwahn, S. 36.

50 De Felip-Jaud, Die Sprichwörter in Hans Vintlers, Pluemen der tugent', S. 272-273.

51 Rabanser, Hexenwahn, S. 36-37.

52 Die „Aberglaubensliste“ Vintlers ist zweigeteilt. Auf 266 Verse folgt eine Bischofslegende, danach behandeln weitere 57 Verse „unchristliche“ Vorstellungen und Gebräuche.

53 Schweitzer, Hans Vintlers "Aberglaubensliste“, S.285-286.

54 Ebd., S. 291-292.

55 Wolfgang Ziegeler, Möglichkeiten der Kritik am Hexen- und Zauberwesen. Zeitgenössische Stimmen und ihre soziale Zugehörigkeit (Kollektive Einstellungen und sozialer Wandel im Mittelalter 2), Köln-Wien 1973, S. 49. 
gelauben an zauberei, / und wissent doch wo da pei / das zauberei got ist unwert. [Vers 7692-7700]“56

"[...] dannoch vindet man ze dieser vrist / die zauberei dannoch phlegen. / etleich die wellen pheil aussegen, / so wellen diese teufel pannen, / das sie bringen guet zesammen. / so wellen etleich warsagen / und wellen vil den teufel fragen, / wa lige golt und edel gestain. / so haben etleich gemain / mit der pösen Erodiana. / so glauben vil an Diana, / di do ain valsche gottin ist, / und etleich mainen haben den list, / das si die leut chunnen schiessen / durch alles gemäur, und etleich giessen / wachs eine pild manigerlai. [Vers 7729-7744]“57

„so nutzen etleich den alraun, / und etleich gelauben an die fraun, / die do haisset Percht mit der eisnen nas. [Vers 7760-7762]“58

"[...] und etleich haben den beibis. / So spricht maniger tummer leib, / die trutte sei ain altes weib / und chumme die leute saugen. [Vers 7795-7798]“59

Im Wertesystem Vintlers sind „abergläubische“ Vorstellungen ein Verstoß gegen die „mässichait". Diesbezügliche Erlebnisse werden von inm auch als teuflische Täuschungen interpretiert und sich darauf einzulassen als Verstoß gegen das erste Gebot des christlichen Glaubens, was Unglauben bzw. Götzendienst bedeute. ${ }^{60}$ Vintler beschrieb auch die objektive Beobachtung, dass während der "var“, also dem Hexenflug, der Körper der „Fahrenden“ an Ort und Stelle verbleibt:

"[...] das sie wänen, sie varen da hin / und mit dem bestrickt sie Sathanas, / das si im gelauben dester pas. / wann wer sich also dem teufel ergeit, / der wänt, er vare alle zeit. / wanne doch der teufel hat / nicht gewalt an chainer stat / hie über des menschen leben, / im welle dann der mensch selben geben. [Vers 8177-8185]“61

Diese Abhandlung über Zauberei behandelt keinen neuen kumulativen Hexenbegriff, sondern gibt Beispiele für verwerfliche alltagsmagische Praktiken. Die eigentliche Kritik Vintlers zielt aber auf Geistliche, welche diese Lehren verbreitet haben sollen, und er fordert ihre Bestrafung:

„auch sprechent si: ,mich hatz gelert / ain pfaff, wie möcht es pös gesein?'/ das sprich ich pei der trewen mein, / das man pilleich ainen solchen pfaffen / darumb sol hertikleichen straffen / das sich zehen stiessen daran, / wann si sein alsampt im pan, / die den gelauben also chrenken [Vers 770 1-7708]“62

56 Ignaz V. Zingerle (Hrsg.), Die Pluemen der Tugent des Hans Vintler, Innsbruck 1874, S. 258-259.

57 Ebd, S. 260.

58 Ebd., S. 261

59 Ebd., S. 262.

60 Schweitzer, Hans Vintlers „Aberglaubensliste“, S. 282-283.

61 Zit. n. Zingerle, Die Pluemen der Tugent, S. 274.

62 Zit. n. Ebd., S. 259. 


\section{Zaubereiprozesse in Tirol}

\section{Erste Verfolgung zauberischer Praktiken in Tirol}

Seit dem 13. Jahrhundert ließen die Tiroler Landesfürsten Rechnungsbücher führen. Dieser Praxis verdanken wir erste Hinweise auf die Verfolgung zauberischer Praktiken, wobei aber oftmals nicht alle Namen der Angeklagten oder deren Urteile erhalten sind. Die älteste diesbezügliche Überlieferung aus dem Jahr 1296 ist in einem Ausgabebetrag des Richters Daniel von Enn mit den Zeilen "pro exustione duarum strigae“ verzeichnet. Die Begriffe "striga” und "malefica” können laut Gabriele Troger bezugnehmend auf Joseph Hansen sowohl mit Hexe als auch mit Giftmörderin gleichgesetzt werden. Sie vermutet daher, wie bereits Hartmann Ammann, dass die beiden "Zauberinnen" im Bozner Unterland Giftmörderinnen waren. ${ }^{63}$

Im Jahr 1371 wurde Obeldein, die Angestellte eines Klosters, wegen Mordversuchs am Probst des Klosters Neustift bei Brixen angeklagt. Das Urteil ist nicht erhalten, jedoch dürfte ein Todesurteil ausgesprochen worden sein. Ihr Mittäter, der Ordensbruder Paul, wurde gegen Leistung der Urfehde freigesprochen. Interessant sind die überlieferten Zaubermittel: Gebete, das Abbrennen von besonderen Kerzen, ein Faden von der Kutte des Probstes und ein durchstochenes Wachsmännchen sowie ein Gemisch aus verbrannten Jungschwalben und Kräutersamen, das dem Probst ins Essen gegeben wurde, sollten diesen töten. ${ }^{64}$

Im Jahr 1433 wurde ein Rechnungsbeleg des Richters Jörg Kel von Enn erstellt, bezüglich der Hinrichtung der "vetter Hannsin und andern die verprannt sind" und der Ausgaben für die „zawbrerin und ander, die gericht sind und die zaubrerin, die man hat gesandt“ . ${ }^{55}$ Neben der gemeinsam mit anderen Personen als Zauberin verbrannten Vetter Hannsin wurde eine weitere Frau genannt, die dem Gericht überstellt wurde. ${ }^{66}$

In Meran wurde 1436/37 eine wegen Zauberei angeklagte Frau freigesprochen: „So ist auf die frawen gangen, die von zauberey wegen von Kastelbell herab geantwort und doch ledig gesprochen worden. ${ }^{\text {"7 } 7}$

Die genannten Verfahren wurden ausnahmslos wegen Zauberei geführt, die charakteristischen Hexerei-Delikte wie Teufelspakt und -buhlschaft, Sabbatfeier und Hexenflug fehlen und sind in Tirol erst ab Anfang des 16. Jahrhunderts zu finden. Auch der "Innsbrucker Hexenprozess" von 1485 drehte sich primär um Zauberei und hat nur ansatzweise die typischen Merkmale eines Hexenprozesses. ${ }^{68}$

63 Gabriele Troger, Der Innsbrucker Hexenprozeß von 1485 als erster und einziger Zaubereiprozeß der inquisition haereticae pravitatis in Tirol, jur. Diss. Innsbruck 1999, S. 8.

64 Rabanser, Hexenwahn, S. 35.

65 TLA, HS 136, Fasc. 28, 29, zit. nach Troger, Der Innsbrucker Hexenprozeß, S. 9.

66 Rabanser, Hexenwahn, S. 196.

67 TLA, HS 200, zit. nach Troger, Der Innsbrucker Hexenprozeß, S. 9.

68 Rabanser, Hexenwahn, S. 35. 


\section{Heinrich Institoris und der Innsbrucker "Hexenprozess“ 1485}

Der Innsbrucker Hexen- bzw. (korrekter bezeichnet) Zaubereiprozess ist der erste und einzige Prozess in Tirol, der nicht vor einem weltlichen Gericht, sondern vor einer kirchlichen Instanz geführt wurde. ${ }^{69}$ Nachdem Heinrich Institoris von Papst Innozenz VIII. die Vollmacht zur inquisitorischen Tätigkeit erhalten hatte, führte der Dominikaner 1485 in Innsbruck den ersten großen Hexenprozess und scheiterte dabei, was aus seiner unmittelbar darauf entstandenen Darstellung im "Hexenhammer" allerdings nicht ersichtlich ist. Ebensowenig wurde der Prozessinhalt darin korrekt wiedergegeben. Anhand der überlieferten Prozessakten kann rekonstruiert werden, dass er eine zeitgenössische Vorstellung von magisch tätigen Frauen, welche sowohl positiven als auch negativen Zauber verrichten konnten, durch den Prozess geschlechtsspezifisch verdichtete. Über fünfzig verdächtigten Frauen standen zwei angeklagte Männer gegenüber, was unter anderem den frauenspezifischen Predigten des Institoris gegen Hexerei geschuldet sein dürfte. ${ }^{70}$ In der Zusammenfassung der Prozessakten durch Hansjörg Rabanser scheinen als Angeklagte nur sieben Frauen auf; dabei handelt es sich um jene, die nach den ersten Zeugenbefragungen am 4. Oktober inhaftiert und in Folge verhört wurden. Die weltlichen Räte und der vom Brixner Bischof Georg Golser zur Kontrolle hinzubeorderte Axamer Pfarrer wurden von Institoris ignoriert und der Prozess nach dessen Gutdünken geführt. Die Anklagepunkte betrafen vor allem Ehebruch, Liebes- und Schadenszauber sowie Mord, aber keine klassischen Hexereidelikte. ${ }^{71}$ In den Denunziationen wurde davon gesprochen, dass die Beschuldigten den Teufel angerufen hätten. Da jedoch in den tatsächlich beschriebenen magischen Praktiken der Name des Teufels nicht vorkommt, ist davon auszugehen, dass Den-Teufel-Anrufen eine gängige Beschreibung für schadenszauberische Tätigkeiten war. $^{72}$

Der Prozess ist vor allem deshalb interessant, weil die Akten eine Momentaufnahme der Übergangsphase von Zaubereiprozessen hin zu Hexereiprozessen bieten. Die Angeklagten wurden hauptsächlich des Schadenszaubers bezichtigt und sind somit eigentlich noch nicht als Hexen, sondern als Zauberinnen zu bezeichnen. Im Gegensatz zu der Vielzahl ländlicher Zaubereivorstellungen, welche sich zu einem großen Teil auf die Schädigung von Vieh, Äckern, das Wettermachen oder Milchdiebstahl bezogen, zeigt dieser Fall einen urbanen Magieschwerpunkt. Zumindest im speziellen Fall wurden fast ausschließlich Liebeszauber und die Anhexung tödlicher Krankheiten angezeigt. Den Hintergrund der Anklage bildete überwiegend ein persönlicher Konflikt zwischen Opfer und denunzierter Person. ${ }^{73}$

69 Rabanser, Hexenwahn, S. 196.

70 Spreitzer, Von den bösen weiben die man nennet die hexen. Frauen und das Böse im Innsbrucker Hexenprozeß und in der dämonologischen Fachprosa im Umkreis Erzherzog Sigmunds, S. 420-421.

71 Rabanser, Hexenwahn, S. 196.

72 Heide Dienst, Lebensbewältigung durch Magie. Alltägliche Zauberei in Innsbruck gegen Ende des 15. Jahrhunderts, in: Alltag im 16. Jahrhundert. Studien zu Lebensformen in mitteleuropäischen Städten, hg. von Alfred Kohler / Heinrich Lutz (Wiener Beiträge zur Geschichte der Neuzeit 14), Wien 1987, S. 80-116, hier S. 106.

73 Spreitzer, Von den bösen weiben, S. 421. 
Neben Nachbarschafts- und Besitzstreitigkeiten, Auseinandersetzungen in Arbeits- oder Mietverhältnissen, sowie innerfamiliären Streitigkeiten wurde der Hexereivorwurf in diesem Fall häufig vor dem Hintergrund des Streits um einen Mann ausgesprochen. Nach vorangegangenen Drohungen der später Denunzierten wurden Auffälligkeiten wie z.B. körperliche Beschwerden der "Opfer“ als kausale Folge der Drohungen interpretiert oder zauberische Handlungen vermutet, welche zum Teil auch tatsächlich stattgefunden haben dürften. Der Glaube an eigene magische Fähigkeiten sowie die Verbreitung von Gerüchten und die konstatierte Wirkungsmacht von Drohungen wurde nach Rainer Walz zum Instrument der Spannungsbewältigung. Die Verdächtigung hatte mehrfach entlastende Funktion: unerklärbare Beschwerden konnten auf ein kausales Motiv zurückgeführt werden und die Ausschaltung der gegnerischen Person forciert sowie eigene Aggressionen projiziert werden. ${ }^{74}$

Der Innsbrucker Hexenprozess kann als Beispiel für die Koevolution zweier Systeme verstanden werden: Bereits vorhandene Deutungsparadigmen konnten durch das Hexenbild der gelehrten Dämonologie stimuliert, kanalisiert und juristisch umgesetzt werden. Institoris stieß auf eine große Bereitschaft zur Denunziation, scheiterte aber vermutlich an der Struktur der Gerüchtebildung, da diese bald zum nicht mehr kontrollierbaren, expansiven Selbstläufer wurde. Die Zahl der Verdächtigungen stieg innerhalb weniger Wochen an, bezog sich schließlich auf über fünfzig Personen, wurde damit zunehmend unglaubwürdiger und erzeugte allgemein Angst, da Magie schließlich eine alltägliche Form der Lebensbewältigung aller Bevölkerungsschichten darstellte. ${ }^{75}$

Das Prozessverfahren wurde nach Beendigung der Verhöre am 21. Oktober von einem eigenen Gerichtshof nachträglich geprüft. In der ersten Sitzung am 29. Oktober plädierte der von bischöflicher Seite eingeschaltete Jurist und Mediziner Johannes Merwais aus Wendingen für die sofortige Freilassung der Angeklagten, er erklärte den Prozess als gesetzwidrig geführt und damit nichtig. Die Nichteinhaltung gültiger Rechtsnormen wurde am 31. Oktober bestätigt und der Prozess für ungültig erklärt. Die sieben Angeklagten wurden unter Eid ihrer Bürgen in den ersten Novembertagen ${ }^{76}$ freigelassen und daraufhin erklärte Bischof Golser die Vollmacht des Inquisitors für erloschen. Der Aufforderung, das Bistum zu verlassen, kam Institoris erst nach mehrmaliger Ermahnung im Feber 1486 nach. ${ }^{77}$

\section{Ein Traktat, das Interesse der Obrigkeit und weitere Prozesse}

Ulrich Molitoris verfasste im Auftrag Erzherzog Sigmunds 1488/89 ein Traktat anlässlich des Prozesses und der Zaubereidiskussion im Land. Obwohl im Text auch von zauberisch und hexerisch tätigen Männern die Rede ist, präsentiert Molitoris die Thematik als ein ausschließlich frauenspezifisches Problem. Die Frage nach der realen Macht der „bösen

74 Spreitzer, Von den bösen weiben, S. 422-423.

75 Ebd., S. 423-424.

76 Als Freilassungsdatum nennt Behringer den 2. November und Rabanser den 3. November. Behringer, Heinrich Kramers "Hexenhammer", S.105-106 u. Rabanser, Hexenwahn, S. 196.

77 Behringer, Heinrich Kramers "Hexenhammer", S.105-106 u. Rabanser, Hexenwahn, S. 196. 
weiben, die man nennen die hexen"78, hinsichtlich Wettermachen und der Verursachung von Krankheiten, Impotenz oder anderen Schäden, wird im Text verneint. Schäden würde der Teufel im Auftrag Gottes den Menschen als Strafe zufügen und die aktive Beteiligung der Hexen sei eine Einbildung, so wie der Hexenflug eine Sinnestäuschung sei. Da Hexen sich aber eine aktive Beteiligung daran zusprächen, damit den für die gesamte Gemeinde schädlichen Zorn Gottes provozieren würden sowie vom Glauben abgefallen wären, forderte Molitoris unter Berufung auf ein Gesetz aus dem Codex Iustiniani die Todestrafe für diese Frauen. ${ }^{79}$ Damit gehört nach Gerd Schwerhoff die Schrift Molitoris' ins "Niemandsland zwischen Wahn und Aufklärung"80.

Interessant in diesem Zusammenhang ist, dass zu Beginn des Jahres 1487 Anna Spiess aus Hall, die frühere Geliebte des Erzherzogs Sigmund, in Innsbruck angeklagt wurde, vier Frauen Schadenszaubereien beigebracht zu haben. Die Grundlage für diese Anklage war eine "Warnung" durch den erzherzoglichen Türhüter Jörg Ött im Auftrag Heinrich Institoris'. Weiters wurde Spiess das Verfassen eines Schreibens angelastet, wonach Erzherzog Sigmunds Ehefrau Katharina von Sachsen und mehrere seiner Räte versucht hätten, den Erzherzog zu vergiften. Die Sache wurde am Landtag in Hall im August 1487 diskutiert und die Beschuldigten im Jänner 1488 des Hochverrats angeklagt. Diese waren jedoch bereits in der ersten Jahreshälfte 1487 geflohen. ${ }^{81}$ Das Interesse des Erzherzogs an Zauberei und magischen Praktiken hatte mehrfach bei Intrigen gegen seine Person eine Rolle gespielt. So hatte man in einem anderen Fall Personen hinter Öfen oder Mauern versteckt, um einen gebannten Teufel zu simulieren, der bestimmte Personen im Beisein Sigmunds denunzierte. Die Betrügereien wurden aufgedeckt, „böse Räte” entlassen und der Landtag stellte den leichtgläubigen Erzherzog de facto unter Kuratel. ${ }^{82}$

Das Interesse der Obrigkeit an magischen Praktiken war durchaus verbreitet. Im April 1496 ließ Kaiser Maximilian in Augsburg ein Schreiben verfassen, in welchem er den Abt von Stams aufforderte, inm jenen Mönch zu schicken, welcher als Geisterbeschwörer tätig sei. ${ }^{83}$ Beim angesprochenen Mönch dürfte es sich um einen Exorzisten gehandelt haben, wobei die Grenze zwischen okkulter Geisterbeschwörung und Teufelsaustreibung eine fließende war. Da Abt Bernhard I. Wälsch antwortete, dass jener Mönch, der Geister bannen konnte, einer von acht Mitbrüdern gewesen sei, welche im vorangegangenen Jahr an einer Seuche verstorben seien, urgierte der Kaiser zumindest die Abschrift eines „Buchle, daraus die bösen Gayst und besessen Leut besworen werden"84, zu erhalten. ${ }^{85}$

78 Ulrich Molitoris, Von den vnholden oder hexen, in: Jörg Mauz, Ulrich Molitoris aus Konstanz (ca. 14421507). Leben und Schriften, Diss. (masch.) Konstanz 1983, S. 339-396, zit. nach Spreitzer, Von den bösen weiben, S. 426.

79 Mauz, Ulrich Molitoris, S. 94 u. 393, zit. nach Ebd., S. 426-427.

80 Gerd Schwerhoff, Rationalität im Wahn. Zum gelehrten Diskurs über die Hexen der frühen Neuzeit, in: Saeculum 37, 1986, S. 65, zit. nach Ebd., S. 428.

81 Rabanser, Hexenwahn, S. 196-197.

82 Ebd., S. 47

83 Ebd, S. 54

84 Abt Stephan Mariacher, Geisterbeschwörer im Stifte Stams (Tiroler Heimatblätter 1 1, 1933), Heft 7/8, S. 277, zit. nach Rabanser, Die Hexenverfolgungen, in: Tirol, S. 96.

85 Rabanser, Hexenwahn, S. 54 u. 197. 
Der "Völser Hexenprozess", welcher die Prozesse im Juli/August 1506 sowie im August 1510 bezeichnet, war der erste Tiroler Prozess, in dem alle klassischen Hexereidelikte genannt wurden. ${ }^{86}$ Insgesamt waren zehn Bäuerinnen und Dienstmägde betroffen. Zwei der Frauen wurden im Sommer 1506 unter Anwendung von Folter befragt. Nach Unterlagen aus dem Jahr 1510 zu schließen wurden diese Frauen hingerichtet. Die Urteile sind nicht erhalten, jedoch dürften auch an den anderen acht Angeklagten Hinrichtungsurteile vollstreckt worden sein. Den erhaltenen Aussagen zufolge hätte der Teufel alle Frauen in psychisch angeschlagenem Zustand aufgesucht und zum Pakt überredet. In Folge wären sie wiederholt zu Hexensabbaten geflogen, hätten gestohlenes Vieh und entwendete Früchte verzehrt, Unzucht getrieben und Unwetter verursacht. Zentrales Thema war auch die Zubereitung und der Verzehr von geraubten Kleinkindern und Ungeborenen, wobei es sich aber um ein irreales magisches Geschehen gehandelt hätte, allerdings wären diese Kinder kurz nach diesen Handlungen verstorben. ${ }^{87}$

Bis ins Jahr 1550 sind weitere 26 Hexerei- und Zaubereiprozesse für den Tiroler Raum

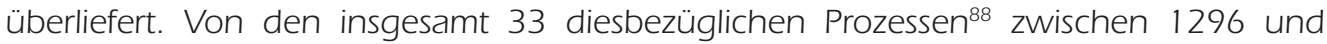
$1550^{89}$ wurden in insgesamt 26 Prozessen Zaubereivergehen verhandelt. Dabei wissen wir von vier Prozessen, in denen ausschließlich gegen Männer verhandelt wurde, in drei Fällen waren beide Geschlechter auf der Anklagebank vertreten und in 19 Prozessen wurden ausnahmslos Frauen angeklagt. Von zehn Männern, denen gesichert ein Prozess gemacht wurde, kamen zwei durch Hinrichtung zu Tode, weitere sieben wurden bestraft, kamen aber mit dem Leben davon. Ein Prozessausgang ist unklar. Mindestens acht Frauen wurden aufgrund einer Zaubereianklage hingerichtet, bei weiteren acht weiblichen Angeklagten ist der Prozessausgang unklar. Die 23 weiblichen Überlebenden mussten in den meisten Fällen die Prozesskosten selbst tragen und wurden zusätzlich des Landes verwiesen. In den sieben Prozessen, in denen Delikte der Hexerei ${ }^{90}$ verhandelt wurden, waren ausschließlich Frauen angeklagt. 14 dieser Frauen wurden hingerichtet, mindestens drei Frauen freigelassen und bei weiteren drei Frauen ist der Prozessausgang unklar. ${ }^{91}$

Die Zaubereiprozesse waren in sich geschlossene Verfahren, während ein Hexenprozess vielfach mindestens einen weiteren nach sich zog, da mittels der Verhöre Denunziationen erreicht wurden. Exemplarisch ist der Fall der im August 1540 in Sarnthein als Hexe angeklagten Barbara Pachler vulgo „Pachlerzottl”. Ihre Aussagen im Verhör führten noch im selben Monat desselben Jahres zu einem Prozess gegen vier Frauen in Stein am Ritten und vermutlich auch zu einem zeitgleichen weiteren Hexenprozess gegen eine

86 Rabanser, Hexenwahn, S. 197-198.

87 Ebd.

88 Die Anfrage Maximilians I. bezüglich des geisterbannenden Mönchs wird bei Rabanser unter den Prozessbiographien aufgelistet, in dieser Aufstellung jedoch nicht berücksichtigt, da auch Rabanser selbst an anderer Stelle die Anfrage als positives Interesse darstellt. Vgl. Rabanser, Hexenwahn, S. 54.

89 Letzteres stellt eine willkürliche Zäsur dar. Sinn der Auflistung ist es zu zeigen, dass auch nach dem ersten Tiroler Hexenprozess weiterhin Zaubereiprozesse stattfanden, dass aber Hexereiprozesse einer anderen Systematik folgten.

90 Der "Innsbrucker Hexenprozess“" wurde in dieser Auflistung zu den Zaubereiprozessen gezählt.

91 Eigene Zusammenfassung anhand der Angaben in: Rabanser, Hexenwahn, S. 198-208. 
unbekannte Anzahl Frauen ${ }^{92}$ in Sarnthein, sowie zu einer Anklage gegen eine Frau in Wangen im Herbst $1540 .{ }^{93}$

Inwieweit die Obrigkeit magischen Vorstellungen anhing, hatte jedenfalls Auswirkungen auf Prozessverläufe. Interessant ist, dass die Überlebenschance bei Zaubereiprozessen in Tirol offensichtlich um ein vielfaches höher war als bei Hexereiprozessen, ebenso war die Anzahl angeklagter Personen pro Prozess wesentlich geringer. ${ }^{94}$ Die höhere Todesrate in Hexereiprozessen ist auch insofern bezeichnend, da in mindestens dreien dieser Prozesse vonseiten der Regierung Unterlagen zur Überprüfung angefordert oder Untersuchungen und Hinrichtungen untersagt wurden. Letzteres wurde im Prozess im August 1540 in Stein am Ritten aber schlichtweg ignoriert, zwei Frauen wurden, anstatt an innen, wie von der Regierung gefordert, die Wirkungslosigkeit von Flugsalben zu demonstrieren, rasch abgeurteilt und verbrannt. Im Herbst desselben Jahres wurde eine in Wangen als Hexe angeklagte Frau durch Intervention der Regierung von mehreren Franziskanern bekehrt und eine Verlautbarung bekanntgegeben, wonach der Bevölkerung beigebracht werden sollte, dass Hexerei und Teufelswerk nur Phantasie und Aberglauben darstellten. ${ }^{95}$

1521 wurde bei einem Prozess in Gries-Bozen eine Frau der Zauberei beschuldigt und von einer diesbezüglich konsultierten Wahrsagerin wurden diverse magische Zaubermittel angegeben, welche in Folge auch aufgefunden wurden. Trotz dieser Vorgehensweise ist keine Intervention von höherer Stelle überliefert. ${ }^{96}$ Als Gegenbeispiel für umfassende magische Glaubensvorstellungen kann der Zaubereiprozess des Jahres 1547 in Sarnthein angeführt werden. Drei bedienstete Frauen hatten am Hof einer magiegläubigen Bäuerin Streiche mit teuflischem Handlungscharakter gespielt, um den Hausbewohnern Angst einzujagen. ${ }^{97}$ Die Anklage lautete auf Zauberei, Betrug, Diebstahl, Untreue gegenüber der Dienstherrenschaft und Kuppelei. Die Hauptangeklagte gestand unter Folter magische Handlungen, die sie zum Teil später widerrief. Die drei Angeklagten wurden unter Berufung auf Alter bzw. Jugend, Einfalt und Armut von Frau Ursula von Sarntal, der Verwalterin der Gerichtsherrnschaft Sarnthein, nur mit dem Landesverweis bestraft. ${ }^{98}$

\section{Schluss}

Zusammenfassend kann festgestellt werden, dass sogenannte "magische“ Praktiken untrennbar mit dem Altag der Menschen des Spätmittelalters und der Neuzeit verbunden waren. Die Intensität der alltagsmagischen Vorstellungen war unabhängig von der sozialen Zugehörigkeit, Inhalt und Praxis des Magieglaubens waren von den unmittelbaren Lebensumständen und Interessen geprägt. Heilmagie und Schutzzauber dienten dem persönlichen Schutz vor konkreten und gedachten Gefahren wie Krankheit

92 Angaben wie "mehrere Unholdinnen“ wurden in der Aufstellung weiter oben als "mindestens zwei" berücksichtigt.

93 Rabanser, Hexenwahn, S. 202

94 Und dies obwohl der "Innsbrucker Hexenprozess" hier als Zaubereiprozess behandelt wurde.

95 Rabanser, Hexenwahn, S. 202-203.

96 Ebd., S. 199

97 Ebd., S. 204

98 Rabanser, Hexenverfolgungen in Tirol, S. 175. 
oder Schadenszauber, der Überwindung persönlicher Probleme und Herausforderungen, sowie der Sicherung von Nahrungsmittelerzeugung und persönlichen Erwerbsquellen. Da magische Praktiken im Sinne eines "do-ut-des" als unbedingt wirksam verstanden wurden, stellten sie eine praktische Ergänzung der Religion hinsichtlich der Bewältigung von Alltagsproblemen dar.

Magische Vorstellungen wurden also nicht in Konkurrenz, sondern als Ergänzung zur kanonisierten Lehre der Amtskirche verstanden und gelebt. Hinzu kam eine Reihe von Praktiken, die als Vermengung kirchlicher Riten und magischer Rituale verstanden werden können, beispielsweise Wetter- oder Flursegen, Reliquienkult und Heiligenverehrung. Ein Großteil der regionalen Geistlichkeit hing ebenfalls magischen Vorstellungen an, eine grundsätzliche Verurteilung passierte vor allem auf höherer theologischer Ebene.

Magische Praktiken zur Alltagsbewältigung, soweit es sich dabei nicht um Schadenszauber handelte, wurden von Obrigkeit und Kirche weitestgehend toleriert, Anschuldigungen bezüglich magischer Verbrechen nicht in allen Fällen verfolgt. Dies erklärt sich daraus, dass der Glaube an Magie kein Schichtenspezifikum darstellte, sondern von großen Teilen der Eliten ebenfalls praktiziert wurde, was auch bedeutete, dass die Bekämpfung von Schadenszaubern mittels Gegenzaubern ein geläufiges und häufig angewandtes Mittel darstellte und es in vielen Fällen gar nicht zu einer Anklage kam. Bei der Verfolgung von Zauberei durch Gerichte und der Bewertung in Traktaten war ausschlaggebend, inwieweit an durch "magische“ Praktiken verursachte Schäden geglaubt wurde oder ob gotteslästerliches Verhalten bestraft werden sollte.

Da davon ausgegangen wurde, dass eine Person, der die Fähigkeit zu zaubern zugeschrieben wurde, gleichermaßen Heil- und Schadensmagie praktizieren konnte, und da letzteres auch durchaus in überzeugter Absicht gelegentlich versucht wurde, war Schuldzuweisung bei "unerklärlichen“ negativen Vorkommnissen eine naheliegende Schlussfolgerung. Genauso dürfte aber auch die Möglichkeit wahrgenommen worden sein, Personen mithilfe eines unterstellten „crimen magiae“ bewusst zu verleumden.

In Abgrenzung zu Hexerei kann davon ausgegangen werden, dass Magie tatsächlich praktiziert wurde und über ihre Wirksamkeit ein weitgehender Konsens geherrscht haben dürfte. Die klassischen Hexereidelikte dürften jedoch kaum wirklich von Personen durchgeführt worden sein, sondern im Wechselspiel von theologischer Dämonologie, alltagsmagischen Vorstellungen und inquisitorischer Verfolgung verbreitet worden sein. ${ }^{99}$ Nicht nur der Glaube an Schadenszauber war verbreitet, sondern sie wurden auch praktiziert, insofern dürften sich diesbezügliche Verurteilungen sowohl auf unterstellte als auch auf real durchgeführte magische Handlungen beziehen.

Intensität und juristische Urteile waren jedoch abhängig von obrigkeitlichen Anschauungen und der Widerständigkeit der Beschuldigten. Im Gegensatz zur Hexerei angeklagten Personen entwickelten sich aus der juristischen Untersuchung eines "crimen magiae" keine Prozessreihen und die Beschuldigten in Tirol hatten statistisch eine 
höhere Überlebenschance. So wurden zwischen 1296 und 1550 im Tiroler Raum 33 diesbezügliche Prozesse verhandelt, wobei in 26 Fällen ein Zaubervergehen behandelt wurde und nur in sieben Fällen zumindest zwei der klassischen Hexereidelikte Gegenstand der Verhandlung waren und somit von einem Hexereiprozess gesprochen werden kann.

Während bei den Zaubereiprozessen in diesem Zeitraum rund ein Fünftel der Angeklagten männlich war, wurden in den Hexereiprozessen ausschließlich Frauen angeklagt. Da nicht alle diese Prozessurteile erhalten sind, können keine absoluten Zahlen genannt werden. Jedoch kann berechnet werden, dass 70-85\% jener Frauen, die in einem Hexereiprozess angeklagt wurden, dabei zu Tode verurteilt wurden. In den genannten Zaubereiprozessen wurden 20-30\% der Männer bzw. rund 21-41\% der Frauen zu Tode verurteilt.

Auch daraus kann abgeleitet werden, dass Schadenszauber aufgrund der gesellschaftlichen Akzeptanz von Zauberei im Allgemeinen als ein weniger schwerwiegendes Delikt verstanden wurde. Die Verfolgungspraxis von magischen Handlungen beruhte auf Denunziation. Bereits vorhandene Deutungsparadigmen wurden von der gelehrten Dämonologie stimuliert. Diesbezügliche theologische Schriften hatten Einfluss auf die Gesetzgebung und vereinfachten damit die Verfolgung magischer Handlungen. Zeitgenössische Kritik, sowohl in Traktaten als auch von Einzelpersonen, hatte zumindest einen mildernden Effekt auf die Bestrafung der angenommenen Verbrechen, jedoch wurden in vielen Fällen schadenszauberische Praktiken schon rein deshalb bestraft, weil sie als Gotteslästerung verstanden wurden.

Die Bandbreite (obrigkeitlicher) Handlungsmöglichkeiten lässt sich exemplarisch am Innsbrucker "Hexenprozess" von 1485 festmachen. Während der Ankläger Heinrich Institoris als überzeugter Inquisitor den Prozess durch Aufrufe zu Denunziationen bewusst initiierte, nutzte der Brixner Bischof Georg Golser die inm zur Verfügung stehende Macht und juristische Grundlagen, um den Prozess zu Fall zu bringen. Insofern muss, unabhängig von einem schichtenübergreifenden Konsens hinsichtlich magischer Vorstellungen und der weiten Verbreitung des Hexereiglaubens, der regionalen geistlichen und weltlichen Obrigkeit eine entscheidende Rolle hinsichtlich Verfolgung von Zaubereiverbrechen zuerkannt werden.

\section{Literatur}

Behringer, Wolfgang, Heinrich Kramers "Hexenhammer“: Text und Kontext, in: Schmaude, Andreas (Hrsg.), Frühe Hexenverfolgung in Ravensburg und am Bodensee (Historische Stadt Ravensburg 21, Konstanz 2001, S. 83-124.

Ders., Hexenverfolgung in Bayern. Volksmagie, Glaubenseifer und Staatsräson in der Frühen Neuzeit, München 1987.

De Felip-Jaud, Elisabeth, Die Sprichwörter in Hans Vintlers ,Pluemen der tugent' und in Tommaso Gozzadinis ,Fiore di Virtù'. Ein Übersetzungsvergleich, in: Gebhardt, Michael/Siller, Max (Hrsg.), Literatur und Sprache in Tirol. Von den Anfängen bis zum 16. Jahrhundert. Akten des 3. Symposiums der Sterzinger Osterspiele (10.-12. April 1995), (Schlernschriften 301), Innsbruck 1996, S. 269-279. 
Dienst, Heide, Lebensbewältigung durch Magie. Alltägliche Zauberei in Innsbruck gegen Ende des 15. Jahrhunderts, in: Kohler, Alfred/Lutz, Heinrich (Hrsg.), Alltag im 16. Jahrhundert. Studien zu Lebensformen in mitteleuropäischen Städten, (Wiener Beiträge zur Geschichte der Neuzeit 14), Wien 1987, S. 80-116.

Dienst, Heide, Magische Vorstellungen und Hexenverfolgungen in den österreichischen Ländern (15. und 18. Jahrhundert), in: Zöllner, Erich (Hrsg.), Wellen der Verfolgung in der österreichischen Geschichte, (Schriften des Institutes für Österreichkunde 48), Wien 1986, S. 70-96.

Dillinger, Johannes, Hexen und Magie. Eine historische Einführung (Historische Einführungen 3), Frankfurt/M. 2007.

Gebhardt, Michael/Siller, Max (Hrsg.), Literatur und Sprache in Tirol. Von den Anfängen bis zum 16. Jahrhundert. Akten des 3. Symposiums der Sterzinger Osterspiele (10.-12. April 1995), (Schlernschriften 301), Innsbruck 1996.

Handle, Stefan, Religiöser und gesellschaftlicher Wandel in Imst in der frühen Neuzeit (Schlernschriften 357), Innsbruck 2013.

Kohler, Alfred/Lutz, Heinrich (Hrsg.), Alltag im 16. Jahrhundert. Studien zu Lebensformen in mitteleuropäischen Städten (Wiener Beiträge zur Geschichte der Neuzeit 14), Wien 1987.

Mariacher, Abt Stephan, Geisterbeschwörer im Stifte Stams, in: Tiroler Heimatblätter 11 (1933), Heft 7/8

Mauz, Jörg, Ulrich Molitoris aus Konstanz (ca. 1442-1507). Leben und Schriften, Diss. (masch.) Konstanz 1983.

Molitoris, Ulrich, Von den vnholden oder hexen, in: Jörg Mauz, Ulrich Molitoris aus Konstanz (ca. 1442-1507). Leben und Schriften, Diss. (masch.) Konstanz 1983, S. 339-396.

Nemec, Helmut, Zauberzeichen. Magie im volkstümlichen Bereich, Wien 1976.

Rabanser, Hansjörg, Die Hexenverfolgungen in Tirol. Verlauf, Prozessbiographien, Interpretation, Diss. Innsbruck 2005.

Ders., Hexenwahn. Schicksale und Hintergründe. Die Tiroler Hexenprozesse, InnsbruckWien 2006.

Schmauder, Andreas (Hrsg.), Frühe Hexenverfolgung in Ravensburg und am Bodensee, (Historische Stadt Ravensburg 2), Konstanz 2001.

Schreiner, Klaus, Laienfrömmigkeit - Frömmigkeit von Eliten oder Frömmigkeit des Volkes? Zur sozialen Verfaßtheit laikaler Frömmigkeitspraxis im späten Mittelalter, in: Schreiner, Klaus (Hrsg.), Laienfrömmigkeit im späten Mittelalter. Formen, Funktionen, politischsoziale Zusammenhänge (Schriften des Historischen Kollegs, Kolloquien 20), MünchenOldenbourg 1992, S. 1-78.

Schreiner, Klaus (Hrsg.), Laienfrömmigkeit im späten Mittelalter. Formen, Funktionen, politisch-soziale Zusammenhänge (Schriften des Historischen Kollegs, Kolloquien 20), München-Oldenbourg 1992. 
Schweitzer, Franz-Josef, Hans Vintlers „Aberglaubensliste“ und der Hexenbegriff, in: Gebhardt, Michael/Siller, Max (Hrsg.), Literatur und Sprache in Tirol. Von den Anfängen bis zum 16. Jahrhundert. Akten des 3. Symposiums der Sterzinger Osterspiele 110.-12. April 1995), (Schlernschriften 301), Innsbruck 1996, S. 281-292.

Schwerhoff, Gerd, Rationalität im Wahn. Zum gelehrten Diskurs über die Hexen der frühen Neuzeit, in: Saeculum 37, 1986.

Spreitzer, Brigitte, Von den bösen weiben die man nennet die hexen. Frauen und das Böse im Innsbrucker Hexenprozeß und in der dämonologischen Fachprosa im Umkreis Erzherzog Sigmunds, in: Literatur und Sprache in Tirol. Von den Anfängen bis zum 16. Jahrhundert. Akten des 3. Symposiums der Sterzinger Osterspiele (10.-12. April 1995), hrsg. von Michael Gebhardt/Max Siller (Schlernschriften 301), Innsbruck 1996, S. 419-431. TLA, HS 136, Fasc. 28.

TLA, HS 200.

Troger, Gabriele, Der Innsbrucker Hexenprozeß von 1485 als erster und einziger Zaubereiprozeß der inquisition haereticae pravitatis in Tirol, jur. Diss. Innsbruck 1999.

Tschacher, Werner, Der Formicarius des Johannes Nider von 1437/38. Studien zu den Anfängen der europäischen Hexenverfolgungen im Spätmittelalter, Aachen 2000.

ULB Innsbruck, Codex 960.

von Hohenheim, Theophrastus, Die große Wundartzney, Frankfurt 1530.

Ziegeler, Wolfgang, Möglichkeiten der Kritik am Hexen- und Zauberwesen. Zeitgenössische Stimmen und ihre soziale Zugehörigkeit (Kollektive Einstellungen und sozialer Wandel im Mittelalter 2), Köln-Wien 1973.

Zingerle, Ignaz V. (Hrsg.), Die Pluemen der Tugent des Hans Vintler, Innsbruck 1874.

Zöllner, Erich (Hrsg.), Wellen der Verfolgung in der österreichischen Geschichte (Schriften des Institutes für Österreichkunde 48), Wien 1986.

Hester Margreiter ist Studentin der Geschichte und der Wirtschaftswissenschaften an der Universität Innsbruck. hester.margreiter@student.uibk.ac.at

\section{Zitation dieses Beitrages}

Hester Margreiter, Magische Alltagsvorstellungen und spätmittelalterliche Zaubereiprozesse in Tirol, in: historia.scribere 7 (2015), S. 409-432, [http://historia.scribere.at], 2014-2015, eingesehen 1.3.2015 (=aktuelles Datum).

(C) Creative Commons Licences 3.0 Österreich unter Wahrung der Urheberrechte der Autorlnnen. 